\title{
The influence of nodule size on the aggressiveness of thyroid carcinoma varies with patient's age
}

\author{
Chaoyang Meng ${ }^{1,2}$, Wenlong Wang ${ }^{1}$, Yuezhong Zhang ${ }^{2}$, Xinying $\mathrm{Li}^{1}$ \\ ${ }^{1}$ Department of General Surgery, Xiangya Hospital, Central South University, Changsha, China; ${ }^{2}$ Xiangya School of Medicine, Central South \\ University, Changsha, China \\ Contributions: (I) Conception and design: X Li, C Meng; (II) Administrative support: X Li; (III) Provision of study materials or patients: C Meng, W \\ Wang, Y Zhang; (IV) Collection and assembly of data: C Meng, W Wang, Y Zhang; (V) Data analysis and interpretation: C Meng, W Wang; (VI) \\ Manuscript writing: All authors; (VII) Final approval of manuscript: All authors. \\ Correspondence to: Xinying Li, MD, PhD, FACS. Department of General Surgery, Xiangya Hospital, Central South University, No.87 Xiangya Road, \\ Changsha, China. Email: lixinyingcn@csu.edu.cn.
}

Background Thyroid nodule size is one of the key parameters that determines the operative approach for thyroid carcinoma. It is necessary to evaluate the influence of nodule size on the aggressiveness of thyroid carcinoma. The eighth edition of staging system has updated the prognostic age cutoff from 45 to 55 years old. It is needed to re-evaluate the difference in aggressiveness of thyroid carcinoma between younger ( $<55$ years old) and older ( $\geq 55$ years old) patients. Importantly, whether the influence of nodule size on the aggressiveness of thyroid carcinoma varies according to the new age stratification remains to be explored.

Methods: Medical records from patients were retrospectively reviewed. Patients with a documented thyroid ultrasonography (US), US-guided fine needle aspiration (FNA) and histopathology were included. The risks of unfavorable events such as central-compartment neck lymph node (CLN) metastasis, lateral-compartment neck lymph node (LLN) metastasis and gross extrathyroidal extension (ETE) were analyzed in four subsets of patients according to size and age.

Results: Large nodule size $(\geq 10 \mathrm{~mm})$ significantly increased the frequencies of CLN metastasis, LLN metastasis and gross ETE $(\mathrm{P}<0.05)$. The frequency of CLN metastasis was significantly higher in younger patients compared with that in older ones. Logistic regression analysis recognized large nodule size as an independent risk factor for all CLN metastasis (OR: 3.304, 95\% CI: 2.473-4.415), LLN metastasis (OR: 9.673, 95\% CI: 4.542-20.597), and gross ETE (OR: 2.430, 95\% CI: 1.508-3.916). Secondly, in younger patients, frequencies of all CLN metastasis, LLN metastasis and gross ETE were significantly higher in nodules $\geq 10 \mathrm{~mm}$ than in nodules $<10 \mathrm{~mm}(\mathrm{P}<0.001)$. However, in older patients, no significant difference was found in the frequencies of LLN metastasis or gross ETE between nodules $<10 \mathrm{~mm}$ and $\geq 10 \mathrm{~mm}$. Logistic regression analysis showed, in younger patients, large nodule size was an independent risk factor for all CLN metastasis (OR: 3.241, 95\% CI: 2.393-4.389), LLN metastasis (OR: 12.495, 95\% CI: 5.281-29.562), and gross ETE (OR: 2.591, 95\% CI: 1.519-4.419), while in older patients large nodule size was recognized as an independent risk factor for CLN metastasis (OR: 3.924, 95\% CI: 1.413-10.899) but not for LLN metastasis or gross ETE.

Conclusions: Large nodule size is significantly related to high aggressiveness of thyroid carcinoma. The correlation between large nodule size and high aggressiveness varies according to patient's age, indicating that the presence of unfavorable events has different clinical significance for patients of varied ages. These findings contribute to accurately assessing the prognosis of individual patient and developing a better management strategy. 
Keywords: Thyroid carcinoma; nodule size; age; extrathyroidal extension; lymph node metastasis

Submitted Oct 07, 2020. Accepted for publication Jan 29, 2021.

doi: $10.21037 / g s-20-747$

View this article at: http://dx.doi.org/10.21037/gs-20-747

\section{Introduction}

Thyroid nodule size plays an important role in making decisions on thyroid carcinoma diagnosis and management. According to 2015 American Thyroid Association (ATA) guidelines, thyroid ultrasound guided fine needle aspiration (US-guided FNA) is strongly recommended to nodules $\geq 10 \mathrm{~mm}$ with intermediate to high suspicion ultrasonography (US) pattern before surgery (1). As for papillary thyroid microcarcinoma (PTMC) which is smaller than $10 \mathrm{~mm}$ in size, a thyroid lobectomy alone is sufficient when patients without prior head or neck radiation, familial thyroid carcinoma, or unfavorable events [such as gross extrathyroidal extension (ETE), central-compartment neck lymph node (CLN) and lateral-compartment neck lymph node (LLN) metastasis] $(2,3)$. However, a near-total or total thyroidectomy can be chosen when nodule size $>10 \mathrm{~mm}$ or patients with unfavorable events (1). Thyroidectomy with modified radical neck dissection is recommended for N1b patients with gross ETE $(1,4)$. Those unfavorable events have been shown to correlate to poor prognosis of patients with thyroid carcinoma, evidenced by increasing recurrence rate at both resection site and regional lymph node, lowering the overall and disease-free survival (5-9). Accordingly, the influence of nodule size on the risks of those unfavorable events should be evaluated.

In addition, patient's age has been shown to have a significant impact on prognosis (3). The frequencies of unfavorable events of patients in different age groups were significantly different (10). Another study also showed the risk of thyroid carcinoma recurrence varied according to patient's age (11). All of these indicate that patient's age is another factor that should be considered when assessing the prognosis of an individual patient and determining management strategy. Recently, the eighth edition of AJCC/UICC modified the prognostic age cutoff from 45 to 55 years old (12). Therefore, it is necessary to re-evaluate whether the risks of prognostic-related unfavorable events are different between patients younger than 55 years old and patients equal to or over 55 years old.

However, risk factors for prognostic-related unfavorable events are hard to be evaluated since multiple factors could affect the evaluation. It has been reported that the risk factors for thyroid carcinoma recurrence were different between patients younger than 45 years and patients equal to or over 45 years old (13). In this scenario, we were wondering whether the impact of nodule size on the risks of unfavorable events varied between patients younger than 55 years old and patients equal to or over 55 years old.

Here, we provided a set of clinical data to demonstrate the influence of thyroid nodule size on the risks of unfavorable events in patients with thyroid carcinoma. We also evaluated the impact of patient's age on the risks of unfavorable events based on the new age stratification updated in the eighth edition of AJCC/UICC staging system. More importantly, different from other studies which only evaluated the influence of patient's age or nodule size on the risk of tumor progression, we further investigated whether the influence of nodule size on the aggressiveness of thyroid carcinoma in patients younger than 55 years old is different from that in patients equal to or over 55 years old.

We present the following article in accordance with the STROBE reporting checklist (available at http://dx.doi. org/10.21037/gs-20-747).

\section{Methods}

\section{Study design}

The study was conducted in accordance with the Declaration of Helsinki (as revised in 2013). The study was approved by the Ethics Committee of Xiangya Hospital Central South University (No. 201912473) and individual consent for this retrospective analysis was waived. Medical records from 2015 through 2018 were retrospectively reviewed. Data from cases which met the inclusion criteria (described in section "Patients inclusion and exclusion") were collected and analyzed. Firstly, the influence of nodule size on the risks of unfavorable events were evaluated in patients with thyroid carcinoma. This study adopted $10 \mathrm{~mm}$ to divide nodules into two groups (nodules $<10 \mathrm{~mm}$ and nodules $\geq 10 \mathrm{~mm}$ ) because: (I) according to 2015 ATA 
guidelines, nodule size $\geq 10 \mathrm{~mm}$ is one of the important factors in deciding to perform US-guided FNA (1); (II) nodule size $<10 \mathrm{~mm}$ is the cutoff value that is widely used to define thyroid microcarcinoma (14-17), which has different prognosis from thyroid carcinoma over $10 \mathrm{~mm}$ in size (18); (III) management strategy may be different between nodules $<10 \mathrm{~mm}$ and nodules $\geq 10 \mathrm{~mm}$, since active surveillance was recommended to low risk papillary microcarcinoma according to studies from Kuma Hospital $(2,19,20)$; (IV) thyroid lobectomy alone is sufficient for thyroid papillary microcarcinoma when patients without risk factors (described in "Introduction" section), while a near-total or total thyroidectomy was recommended when nodule size $>10 \mathrm{~mm}$ as well as patients with risk factors (1). The risks of unfavorable events were compared between these two groups. Secondly, the risks of unfavorable events were re-evaluated based on age stratification where 55 years old was chosen as the cutoff value according to the eighth edition of AJCC/UICC staging system. Thirdly, whether the influence of nodule size on the aggressiveness of thyroid carcinoma varies with patient's age was investigated.

\section{Patients inclusion and exclusion}

Patients with all documented thyroid US, US-guided FNA records and surgical histopathology were collected. Such nodules were excluded: nodules with incomplete information or without formal US report; patient's records without a clear correlation in nodular location, cytology, and histopathology; patients with an incidental thyroid cancer (defined as a malignant lesion found outside the nodule of interest).

\section{Treatment}

Lobectomy or thyroidectomy with or without neck lymph node dissection was chosen based on 2015 ATA guidelines. Prophylactic CLN dissection (ipsilateral or bilateral) was performed when nodules $>40 \mathrm{~mm}$ or lymph node enlargement was observed under US. LLN dissection was performed for patients with suspicious LLN metastasis evaluated by US and computerized tomography scan or US-guided FNA proven-metastatic lateral neck lymphadenopathy.

\section{FNA technique and categorization}

Thyroid FNA at our hospital was performed under US guidance and by the same surgical team. All FNA performers received certification and support from the cytology and ultrasound technician. The final FNA results were verified by at least one experienced cytopathologist (21). Nodules smaller than $10 \mathrm{~mm}$ in size would receive US-guided FNA when at least one of the following requirements was met: Nodular location was close to thyroid capsule, trachea, or nerve; Patients had a family history of thyroid cancer or a history of radiotherapy; Enlargement of neck LN was observed by US or neck LN was suspicious of cancer metastasis. Patients had a heavy psychological burden and strongly demanded US-guided FNA to evaluate nodular pathological type. US-guided FNA would be recommended to nodules equal to or larger than $10 \mathrm{~mm}$ in size when at least one of the following requirements was met: TIRADS 4c-6, TI-RADS 4b and nodule size $\geq 15 \mathrm{~mm}$, TIRADS $4 \mathrm{a}$ and nodule size $\geq 25 \mathrm{~mm}$, TI-RADS 3 and nodule size $\geq 40 \mathrm{~mm}$, or any nodule that needed thyroidectomy. If a patient had more than one biopsy on the same nodule, the FNA record immediately before surgery was adopted in the following analysis. Cytology results were recorded as nondiagnostic or unsatisfactory (Bethesda I), benign (Bethesda II), atypical (atypia of undetermined significance and follicular lesion of undetermined significance, Bethesda III), follicular neoplasm/suspicious for follicular neoplasm (Bethesda IV), suspicious for malignancy (Bethesda V), or malignancy (Bethesda VI) according to the 2009 Bethesda System for Reporting Thyroid Cytopathology (22).

\section{Clinical data acquisition}

The electronic medical records were reviewed to collect clinical data, including age, gender, US report, USguided FNA records, and histopathology. Neck lymph node metastasis was confirmed by histopathology. Gross extrathyroidal extension was identified during surgery and confirmed by histopathology. Nodule size was described in terms of maximum diameter measured by US. Kwak's Thyroid Imaging Reporting and Data System (TI-RADS) was used in our hospital (23). Five suspicious signs of thyroid cancer were included in this risk stratification system, namely solid composition with hypoechogenicity, taller than wide in shape, irregular margin or ETE, microcalcification, and abnormal neck LN. Nodules with one, two, three/four, or five suspicious signs were classified as TI-RADS 4a, 4b, 4c, and 5, respectively. Nodules with cancer cells in cytology were classified as TI-RADS 6. 


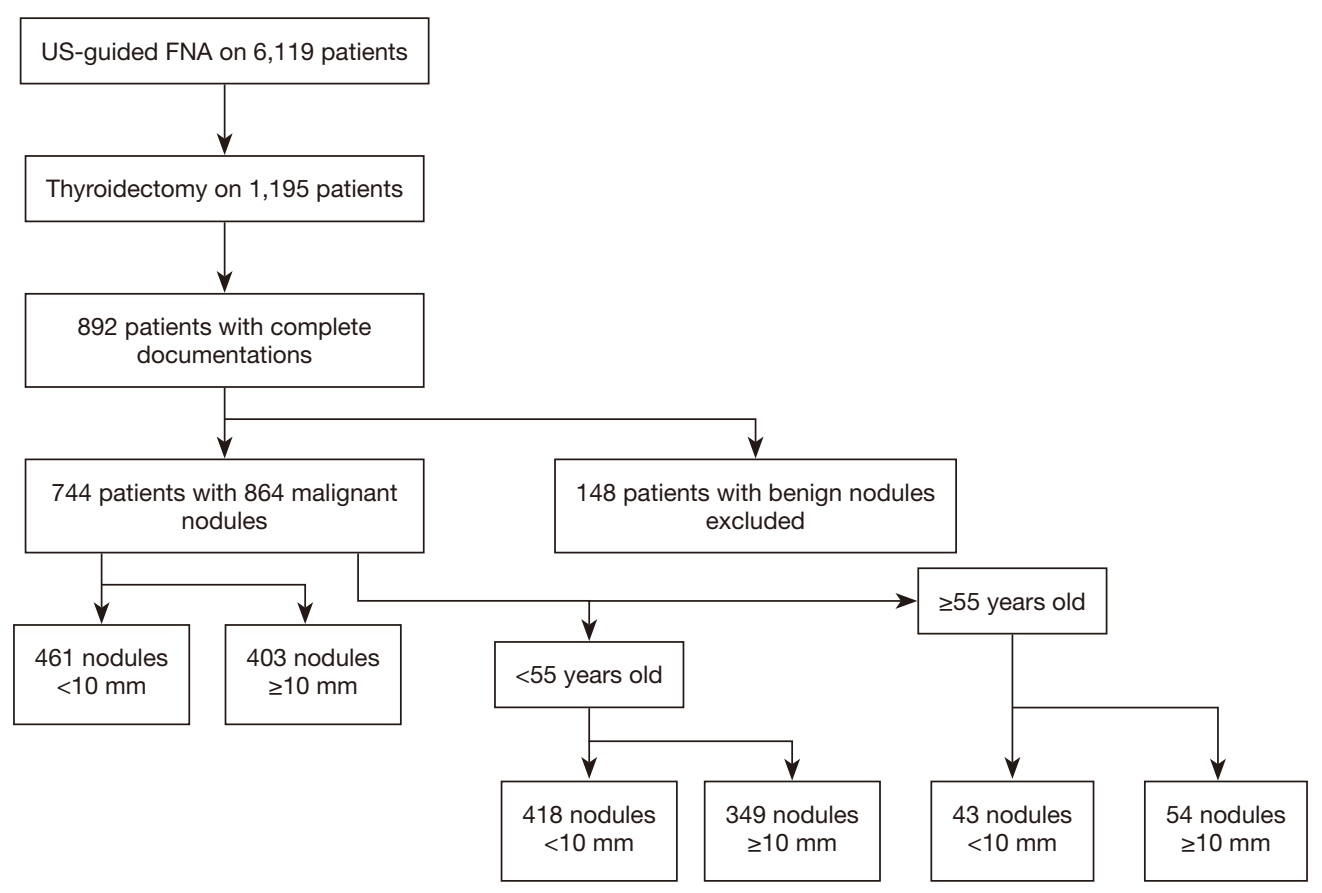

Figure 1 Flow chart of study patients. US-guided FNA, ultrasound guided fine needle aspiration.

\section{Statistical analysis}

Continuous data were presented as medians with percentiles $(25 \%$ and $75 \%)$ if they were not normally distributed (determined by the Shapiro-Wilk test), and groups were compared using Wilcoxon rank-sum test. Categorical data were analyzed using chi-square test (Fisher's exact test). Logistic regression analysis was used for independent risk factor analysis. Since all variables were recorded in the ultrasound report, US-guided FNA report or histopathology report, no data was missing for all patients. Patients with incomplete documentation were excluded. Data were analyzed using SPSS (v.23; SPSS/IBM) for Windows. A two-sided $\mathrm{P}<0.05$ was considered significant.

\section{Results}

\section{Clinical characteristics}

US-guided FNA was performed on 6,119 patients, of which surgery was performed on 1,195 patients. Final diagnosis was determined by histopathology. After excluding patients that did not meet the inclusion criteria, there were 1,012 nodules from 892 patients included in this study (Figure 1). The overall median of age was 42 years old. The overall median of nodule size was $9 \mathrm{~mm}$. In 1,012 nodules, $1.1 \%$ were diagnosed as ND/USF with malignancy ratio of $18.2 \%, 4.9 \%$ as benign with malignancy ratio of $28.0 \%$, $8.8 \%$ as AUS/FLUS with malignancy ratio of $55.1 \%, 0.7 \%$ as FN/SFN with malignancy ratio of $14.3 \%, 19.5 \%$ as SM with malignancy ratio of $79.2 \%$, and $65.0 \%$ as malignant with malignancy ratio of $97.6 \%$. CLN metastasis happened to 356 patients, LLN metastasis occurred in 71 patients, and gross ETE happened to 86 patients. Other statistical results were presented in Table 1. Of note, 864 out of 1,012 nodules were malignant and participated in the following analysis.

\section{Both large nodule size and age younger than 55 years increased the risk of CLN metastasis}

Unfavorable events occurred in thyroid carcinoma such as gross ETE and neck LN metastasis have a significant impact on patients' prognosis $(5,8,24)$. Hence, the frequencies of CLN metastasis, LLN metastasis and gross ETE were analyzed and compared in nodules $<10 \mathrm{~mm}$ and $\geq 10 \mathrm{~mm}$ (Table 2). The frequencies of all CLN metastasis, LLN metastasis and gross ETE were significantly higher in nodules $\geq 10 \mathrm{~mm}$ compared to those in nodules $<10 \mathrm{~mm}$ $(\mathrm{P}<0.05)$ : almost twice as high in CLN metastasis $(\mathrm{P}<0.001)$, over 9 times as high in LLN metastasis $(\mathrm{P}<0.001)$, and 
Table 1 Clinical characteristics

\begin{tabular}{|c|c|c|c|c|}
\hline Characteristics & \multicolumn{2}{|c|}{ Benign } & \multicolumn{2}{|c|}{ Malignant } \\
\hline Age, years & 48 & $38,53^{*}$ & 42 & $33,50^{\star}$ \\
\hline Size, mm & 10 & $6,21.5^{\star}$ & 9 & $6,15^{\star}$ \\
\hline \multicolumn{5}{|l|}{ Gender } \\
\hline Female & 124 & 15.5 & 677 & 84.5 \\
\hline \multicolumn{5}{|l|}{ TI-RADS } \\
\hline 3 & 39 & 45.3 & 47 & 54.7 \\
\hline $4 a$ & 74 & 16.2 & 382 & 83.8 \\
\hline 5 & 0 & 0 & 38 & 100 \\
\hline 6 & 0 & 0 & 3 & 100 \\
\hline \multicolumn{5}{|l|}{ Bethesda } \\
\hline I & 9 & 81.8 & 2 & 18.2 \\
\hline ॥ & 36 & 72.0 & 14 & 28.0 \\
\hline III & 40 & 44.9 & 49 & 55.1 \\
\hline IV & 6 & 85.7 & 1 & 14.3 \\
\hline V & 41 & 20.8 & 156 & 79.2 \\
\hline No & 148 & 15.7 & 793 & 84.3 \\
\hline Yes & 0 & 0 & 71 & 100 \\
\hline \multicolumn{5}{|l|}{ ETE } \\
\hline No & 148 & 16.0 & 778 & 84.0 \\
\hline Yes & 0 & 0 & 86 & 100 \\
\hline
\end{tabular}

*, the percentiles of $25 \%$ and $75 \%$. TI-RADS, thyroid imaging reporting and data system; CLN-meta, central-compartment neck lymph node metastasis; LLN-meta, lateral-compartment neck lymph node metastasis; ETE, gross extrathyroidal extension.

over twice as high in gross ETE $(\mathrm{P}<0.001)$. Besides, the frequency of nodules with high TI-RADS score (TI-RADS $4 \mathrm{c}-6)$ significantly increased in nodules $\geq 10 \mathrm{~mm}$ compared to that in nodules $<10 \mathrm{~mm}(18.1 \%$ vs. $10.8 \%, \mathrm{P}=0.002)$.

Patient's age has been shown to be related to prognosis (25).
The frequencies of CLN metastasis, LLN metastasis and gross ETE were investigated between patients $<55$ years old and patients $\geq 55$ years old. There was no significant difference in the frequency of LLN metastasis or gross ETE. However, CLN metastasis happened more frequently 
Table 2 Comparison of the frequencies of CLN metastasis, LLN metastasis and ETE between nodules $<10 \mathrm{~mm}$ and $\geq 10 \mathrm{~mm}$

\begin{tabular}{|c|c|c|c|c|c|c|c|c|c|c|c|c|}
\hline $\begin{array}{l}\text { Size, } \\
\mathrm{mm}\end{array}$ & \multicolumn{3}{|c|}{ TIRADS score $\geq 4 c$} & \multicolumn{3}{|c|}{ CLN-meta } & \multicolumn{3}{|c|}{ LLN-meta } & \multicolumn{3}{|c|}{ Gross ETE } \\
\hline$<10$ & $50 / 10.8$ & 0.002 & $\begin{array}{c}1.818 \\
(1.234,2.680)\end{array}$ & $133 / 28.9$ & $<0.001$ & $\begin{array}{c}3.055 \\
(2.306,4.048)\end{array}$ & 8/1.7 & $<0.001$ & $\begin{array}{c}10.492 \\
(4.961,22.189)\end{array}$ & $28 / 6.1$ & $<0.001$ & $\begin{array}{c}2.600 \\
(1.621,4.171)\end{array}$ \\
\hline$\geq 10$ & 73/18.1 & & & $223 / 55.3$ & & & $63 / 15.6$ & & & $58 / 14.4$ & & \\
\hline
\end{tabular}

TI-RADS, thyroid imaging reporting and data system; CLN-meta, central-compartment neck lymph node metastasis; LLN-meta, lateral-compartment neck lymph node metastasis; ETE, gross extrathyroidal extension; OR, odd ratio; CI, confidence interval.

Table 3 Comparison of the frequencies of CLN metastasis, LLN metastasis and ETE between patients $<55$ years old and patients $\geq 55$ years old

\begin{tabular}{|c|c|c|c|c|c|c|c|}
\hline Age, years & \multicolumn{3}{|c|}{ CLN-meta (count/ratio) } & \multicolumn{2}{|c|}{ LLN-meta } & \multicolumn{2}{|c|}{ Gross ETE } \\
\hline$<55$ & $331 / 37.4$ & $<0.001$ & $2.239(1.433,3.497)$ & $63 / 7.1$ & 0.717 & $73 / 8.3$ & 0.062 \\
\hline$\geq 55$ & $27 / 21.1$ & & & $8 / 6.3$ & & $17 / 13.3$ & \\
\hline
\end{tabular}

CLN-meta, central-compartment neck lymph node metastasis; LLN-meta, lateral-compartment neck lymph node metastasis; ETE, gross extrathyroidal extension; OR, odd ratio; $\mathrm{Cl}$, confidence interval.

in patients younger than 55 years (Table 3).

And then, risk factors for unfavorable events were analyzed through logistic analysis (Table 4). Large nodule size $(\geq 10 \mathrm{~mm})$ was recognized as an independent risk factor for CLN metastasis (OR: 3.304, 95\% CI: 2.473-4.415), LLN metastasis (OR: 9.673, 95\% CI: 4.542-20.597), and gross ETE (OR: 2.430, 95\% CI: 1.508-3.916). In addition to large nodule size, age younger than 55 years (OR: 1.947, 95\% CI: 1.221-3.105) and male gender (OR: 2.112, 95\% CI: $1.497-2.980)$ were recognized as another two independent risk factors for CLN metastasis, while high TI-RADS score (TI-RADS 4c-6, OR: 4.483, $95 \%$ CI: 2.594-7.749) was recognized as another independent risk factor for LLN metastasis and gross ETE. Interestingly, age younger than 55 years was recognized as a protective factor for gross ETE (OR: 0.465, 95\% CI: 0.261-0.830).

\section{The impact of nodule size on the risks of unfavorable events varies according to age stratification}

To explore whether the influence of nodule size on the risks of prognostic-related unfavorable events correlate to patient's age, the frequencies of unfavorable events in patients with nodules $<10 \mathrm{~mm}$ and $\geq 10 \mathrm{~mm}$ were compared based on age stratification. In patients younger than 55 years, frequencies of all CLN metastasis, LLN metastasis and gross ETE were significantly higher in nodules $\geq 10 \mathrm{~mm}$ than in nodules $<10 \mathrm{~mm}(\mathrm{P}<0.001)$. However, in older patients, there was no significant difference in the frequencies of LLN metastasis or gross ETE but only in the frequency of CLN metastasis (Table 5). Besides, in patients younger than 55 years logistic regression analysis recognized large nodule size as an independent risk factor for all CLN metastasis (OR: 3.241, 95\% CI: 2.393-4.389), LLN metastasis (OR: 12.495, 95\% CI: 5.281-29.562), and gross ETE (OR: 2.591, 95\% CI: 1.519-4.419). By contrast, in older patients, large nodule size was recognized as an independent risk factor for only CLN metastasis (OR: 3.924, 95\% CI: 1.413-10.899) but not for LLN metastasis or gross ETE (Table 6). The logistic regression analysis also showed, in patients younger than 55 years, male gender was an independent risk factor for CLN metastasis while high TI-RADS score was an independent risk factor for LLN metastasis and gross ETE. However, in older patients, large nodule size was the only one risk factor for CLN metastasis and high TI-RADS score was the only one risk factor for LLN metastasis, and no risk factor was recognized for gross ETE.

\section{Discussion}

In our hospital, Kwak's TI-RADS was adopted to characterize thyroid nodules as described in section "FNA 
Table 4 Risk factors for CLN metastasis, LLN metastasis and ETE.

\begin{tabular}{|c|c|c|c|c|c|c|c|c|c|}
\hline Factors & \multicolumn{3}{|c|}{ CLN-meta } & \multicolumn{3}{|c|}{ LLN-meta } & \multicolumn{3}{|c|}{ Gross ETE } \\
\hline \multicolumn{10}{|c|}{ Age, years } \\
\hline$\geq 55$ & $27 / 27.8$ & & & $8 / 8.2$ & 0.991 & & $17 / 17.5$ & 0.008 & $\begin{array}{c}0.465 \\
(0.261,0.830)\end{array}$ \\
\hline \multicolumn{10}{|l|}{ Gender } \\
\hline Male & $102 / 54.5$ & $<0.001$ & $\begin{array}{c}2.112 \\
(1.497,2.980)\end{array}$ & $20 / 10.7$ & 0.302 & & $17 / 9.1$ & 0.505 & \\
\hline Female & $254 / 37.5$ & & & $51 / 7.5$ & & & $69 / 10.2$ & & \\
\hline$\geq 10$ & $223 / 55.3$ & $<0.001$ & $\begin{array}{c}3.304 \\
(2.473,4.415)\end{array}$ & $63 / 15.6$ & $<0.001$ & $\begin{array}{c}9.673 \\
(4.542,20.597)\end{array}$ & $58 / 14.4$ & $<0.001$ & $\begin{array}{c}2.430 \\
(1.508,3.916)\end{array}$ \\
\hline \multicolumn{10}{|l|}{ TI-RADS } \\
\hline $3-4 b$ & $292 / 39.4$ & 0.130 & & $42 / 5.7$ & & & $62 / 8.4$ & & \\
\hline $4 c-6$ & $64 / 52.0$ & & & 29/23.6 & $<0.001$ & $\begin{array}{c}4.483 \\
(2.594,7.749)\end{array}$ & $24 / 19.5$ & 0.001 & $\begin{array}{c}2.384 \\
(1.412,4.027)\end{array}$ \\
\hline
\end{tabular}

TI-RADS, thyroid imaging reporting and data system; CLN-meta, central-compartment neck lymph node metastasis; LLN-meta, lateralcompartment neck lymph node metastasis; ETE, gross extrathyroidal extension; OR, odd ratio; Cl, confidence interval.

Table 5 Comparison of the frequencies of CLN metastasis, LLN metastasis and ETE between nodules $<10 \mathrm{~mm}$ and $\geq 10 \mathrm{~mm}$ based on age stratification.

\begin{tabular}{|c|c|c|c|c|c|c|}
\hline Age, years & \multicolumn{2}{|c|}{ CLN-meta } & \multicolumn{2}{|c|}{ LLN-meta } & \multicolumn{2}{|c|}{ Macro-ETE } \\
\hline \multicolumn{7}{|l|}{$<55$} \\
\hline Nodule $<10 \mathrm{~mm}$ & $127 / 30.4$ & $<0.001$ & $6 / 1.4$ & $<0.001$ & $22 / 5.3$ & $<0.001$ \\
\hline Nodule $\geq 10 \mathrm{~mm}$ & $202 / 57.9$ & & $57 / 16.3$ & & $47 / 13.5$ & \\
\hline \multicolumn{7}{|l|}{$\geq 55$} \\
\hline Nodule $<10 \mathrm{~mm}$ & $6 / 14.0$ & 0.006 & $2 / 4.7$ & 0.251 & $6 / 14.0$ & 0.409 \\
\hline Nodule $\geq 10 \mathrm{~mm}$ & $21 / 38.9$ & & $6 / 11.1$ & & $11 / 20.4$ & \\
\hline
\end{tabular}

CLN-meta, central-compartment neck lymph node metastasis; LLN-meta, lateral-compartment neck lymph node metastasis; ETE, gross extrathyroidal extension.

technique and categorization". The ratio of malignancy in the current study was $54.7 \%$ in TI-RADS $3,83.8 \%$ in TIRADS $4 \mathrm{a}, 92.3 \%$ in TI-RADS $4 \mathrm{~b}, 90.1 \%$ in TI-RADS $4 \mathrm{c}$, and $100 \%$ in TI-RADS 5 and 6 . Some nodules were not included into the current study because nodules with low TI-RADS score (TI-RADS 3-4b) did not receive USguided FNA or some Bethesda I-III nodules did not receive surgery, causing the ratio of malignancy in TI-RADS 3-4b was over-estimated. The report of US-guided FNA has six categories according to Bethesda System for Reporting 
Table 6 Risk factors for CLN metastasis, LLN metastasis and ETE based on age stratification.

\begin{tabular}{|c|c|c|c|c|c|c|}
\hline Age, years & \multicolumn{2}{|r|}{ CLN-meta } & \multicolumn{2}{|r|}{ LLN-meta } & \multicolumn{2}{|c|}{ Macro-ETE } \\
\hline \multicolumn{7}{|l|}{$<55$} \\
\hline \multicolumn{7}{|l|}{ Gender } \\
\hline Male & $<0.001$ & $2.365(1.641,3.408)$ & 0.334 & & 0.366 & \\
\hline \multicolumn{7}{|l|}{ Size } \\
\hline \multicolumn{7}{|l|}{$<10 \mathrm{~mm}$} \\
\hline$\geq 10 \mathrm{~mm}$ & $<0.001$ & $3.241(2.393,4.389)$ & $<0.001$ & $12.495(5.281,29.562)$ & $<0.001$ & $2.591(1.519,4.419)$ \\
\hline \multicolumn{7}{|l|}{ TI-RADS } \\
\hline \multicolumn{7}{|l|}{$\geq 55$} \\
\hline \multicolumn{7}{|l|}{ Gender } \\
\hline Male & 0.804 & & 0.773 & & 0.836 & \\
\hline \multicolumn{7}{|l|}{ Female } \\
\hline \multicolumn{7}{|l|}{ Size } \\
\hline \multicolumn{7}{|l|}{$<10 \mathrm{~mm}$} \\
\hline$\geq 10 \mathrm{~mm}$ & 0.009 & $3.924(1.413,10.899)$ & 0.436 & & 0.409 & \\
\hline \multicolumn{7}{|l|}{ TI-RADS } \\
\hline
\end{tabular}

TI-RADS, thyroid imaging reporting and data system; CLN-meta, central-compartment neck lymph node metastasis; LLN-meta, lateral-compartment neck lymph node metastasis; ETE, gross extrathyroidal extension; OR, odd ratio; Cl, confidence interval.

Thyroid Cytopathology (22). Repeated US-guided FNA is recommended when Bethesda I or III is reported. Bethesda II nodules will flow to clinical follow-up, while Bethesda IV-VI nodules are recommended to lobectomy or thyroidectomy. The current study investigated 1,012 nodules, including $11(1.09 \%)$ Bethesda I nodules, 50 (4.94\%) Bethesda II nodules and 89 (8.79\%) Bethesda III nodules. The reasons for performing surgery on these nodules were as the followings: contralateral nodule was malignant; nodule size was over $30 \mathrm{~mm}$ and aesthetic problems occurred; Bethesda III was reported in repeated US-guided FNA as well as the nodule with high TI-RADS score ( $>4 \mathrm{a})$; patient bore a heavy psychological burden and demanded surgery. As surgery is not recommended to Bethesda I-III nodules according to 2015 ATA guidelines, a considerable proportion of Bethesda I-III nodules were not included in this study, resulting in overestimation of the malignant ratio in Bethesda I-III nodules in this study.

Overdiagnosis and overtreatment are two main concerns in regard to the management of thyroid sub-centimeter nodules. Studies have shown that most PTMC are in an inert state, and it is not too late to perform surgery after disease progression during active surveillance $(2,3)$. However, active surveillance rather than immediate surgery over sub-centimeter nodules has not yet reached a consensus. Researchers have found that thyroidectomy on patients with thyroid tumors $<10 \mathrm{~mm}$ were safer and involved fewer postoperative complications compared to that on patients with tumors $\geq 10 \mathrm{~mm}$ (18). Patients who suffered from differentiated thyroid microcarcinoma and 
received immediate treatment had superior prognosis than those who received surgery when tumor size $\geq 10 \mathrm{~mm}$, evidenced by higher disease-free survival (18). Patients for active surveillance should be carefully selected. In Miyauchi and colleagues' studies, only patients without unfavorable events were included in observation cohort $(2,3)$. It is worth noting that all patients in observation cohort received US-guided FNA to exclude high-grade malignancy, since there is no way to confidently predict which tumor may undergo a more aggressive process (26). Therefore, aggressive types of thyroid carcinoma would be missed due to similar US pattern without US-guided FNA $(27,28)$. It has been described by ATA guidelines that locally invasive primary tumors may be associated with symptoms including progressive dysphagia, respiratory compromise, hemoptysis, significant voice change or the finding of vocal cord paralysis, and mass fixation to the airway or neck structures.

It has been shown that unfavorable events correlated to poor prognosis. ETE associated with lower disease-free or diseasespecific survival, higher recurrence rate at both resection site and regional lymph node in patients with PTC (5-7), even microscopic ETE (identified by definite histology) was related to lower relapse-free survival (8). Furthermore, the extent of ETE was considered as a significant determinant of prognosis in patients with PTC, evidenced by a five-time increase in the risk of recurrence in patients with multiple structures invaded by tumor cells in comparison with patients with single invaded structure (29). In patients with initial distant metastasis when diagnosed, ETE can further result in poorer prognosis compared to that in those without initial metastasis, characterized by a shorter time period from surgery to tumor progression, lower disease-specific survival, or no remission after surgery (30). Neck LN metastasis has also been recognized as a significant predictor of poor overall survival (9). The overall survival decreased from $82 \%$ to $79 \%$ when neck LN metastasis occurred. In PTC patients older than 45 years, neck LN metastasis dramatically increased the risk of death by $46 \%$ (31). Another study on patients younger than 45 years showed that the mortality risk was not only associated with $\mathrm{LN}$ metastasis, but also correlated with the number of metastatic LN, namely incrementally more metastatic lymph nodes up to six conferred additional risk of death (24). Therefore, the relationship between nodule size and the risks of unfavorable events was investigated in the current study. Our results showed that large nodule size $(\geq 10 \mathrm{~mm}$ ) significantly increased the risk of CLN metastasis, LLN metastasis and gross ETE. Consequently, small tumor size at diagnosis and treatment is contributed to lowering the incidences of unfavorable events and possibly improving patients' prognosis.

In addition, patient's age has been reported to be related to prognosis. The growth activity of tumor in subclinical papillary thyroid microcarcinoma significantly decreased with patient age (32). Young age ( $<40$ years) was recognized as an independent predictor of tumor progression (32). A separate study reported that LN metastasis more frequently occurred in patients younger than 50 years (10). In this current study, younger patients were defined as $<55$ years according to the eighth edition of AJCC/UICC staging system (12). The frequency of CLN metastasis was significant higher in younger patients compared with older ones. However, there was no significant difference in the frequency of LLN metastasis or gross ETE. Besides, we also investigated the risk factors for different unfavorable events. It was found that age younger than 55 years was a risk factor for CLN metastasis but not for LLN metastasis. Interestingly, age equal to or older than 55 years was a risk factor for gross ETE. This is possibly due to a longer period of tumor growth in older patients. Male gender was a risk factor for CLN metastasis but not for LLN metastasis or gross ETE.

Patient's age has been reported to influence the risk factors for recurrence. Male gender and multifocality were recognized as the independent risk factors for thyroid carcinoma recurrence in patients younger than 45 years old, while lymph node metastasis and multifocality were the risk factors in patients equal to or older than 45 years (13). Therefore, we evaluated for the first time whether the influence of nodule size on the risks of unfavorable events are varied between younger patients and older patients. It was found that, in patients younger than 55 years, nodule size $\geq 10 \mathrm{~mm}$ was one of the independent risk factors for all CLN metastasis, LLN metastasis and gross ETE. However, in older patients, large nodule size would not increase the risks of LLN metastasis or gross ETE. Consistently, this current study revealed that the composition of risk factors for unfavorable events are different between younger patients and older ones. In younger patients, male gender was recognized as another risk factor for CLN metastasis, while high TI-RADS score (TI-RADS 4c-6) was another risk factor for LLN metastasis and gross ETE. However, in older patients, large nodule size was recognized as the only one risk factor for CLN metastasis and high TIRADS score was the only one risk factor for LLN metastasis, and no risk factor was recognized for gross ETE. These results indicate that larger malignant nodules in younger 
patients display higher aggressiveness. Therefore, earlier treatment is recommended to young patients with thyroid carcinoma.

\section{Limitations}

Selection bias exist in this study, as US-guided FNA is not recommended to small nodules $(<10 \mathrm{~mm})$ and surgery is not recommended to Bethesda I-III nodules. In this context, the risks of unfavorable events in the group with nodules $<10 \mathrm{~mm}$ was over-estimated, which in turn underestimated the difference in the risks of unfavorable events between the group with nodules $<10 \mathrm{~mm}$ and the group with nodules $\geq 10 \mathrm{~mm}$.

\section{Conclusions}

Large nodule size significantly increases the aggressiveness of thyroid carcinoma. This impact varied according to patient's age: in patients younger than 55 years, large nodule size $(\geq 10 \mathrm{~mm})$ increases the risk of CLN metastasis, LLN metastasis and gross ETE, while in older patients, large nodule size only increases the risk of CLN metastasis. These results indicate that the presence of unfavorable events has different clinical significance for patients of varied ages. This finding contributes to assessing the prognosis of individual patient and developing a better management strategy.

\section{Acknowledgments}

The authors would like to acknowledge Linlin Wan (Neurology Department, Xiangya Hospital, Central South University) who contributed a lot in collecting data for the present study and Shuyue Zhou (Houston Methodist Research Institute, Texas, United States) who polished the language.

Funding: This work is supported by the National Natural Science Foundation (NSFC) of China (No. 82073262).

\section{Footnote}

Reporting Checklist: The authors have completed the STROBE reporting checklist. Available at http://dx.doi. org/10.21037/gs-20-747

Data Sharing Statement: Available at http://dx.doi. org/10.21037/gs-20-747
Conflicts of Interest: All authors have completed the ICMJE uniform disclosure form (available at http://dx.doi. org/10.21037/gs-20-747). XL serves as an unpaid Associate Editor of Gland Surgery from Mar 2013 to Feb 2023. The other authors have no conflicts of interest to declare.

Ethical Statement: The authors are accountable for all aspects of the work in ensuring that questions related to the accuracy or integrity of any part of the work are appropriately investigated and resolved. The study was conducted in accordance with the Declaration of Helsinki (as revised in 2013). The study was approved by the Ethics Committee of Xiangya Hospital Central South University (No. 201912473) and individual consent for this retrospective analysis was waived.

Open Access Statement: This is an Open Access article distributed in accordance with the Creative Commons Attribution-NonCommercial-NoDerivs 4.0 International License (CC BY-NC-ND 4.0), which permits the noncommercial replication and distribution of the article with the strict proviso that no changes or edits are made and the original work is properly cited (including links to both the formal publication through the relevant DOI and the license). See: https://creativecommons.org/licenses/bync-nd/4.0/.

\section{References}

1. Haugen BR, Alexander EK, Bible KC, et al. 2015 American Thyroid Association Management Guidelines for Adult Patients with Thyroid Nodules and Differentiated Thyroid Cancer: The American Thyroid Association Guidelines Task Force on Thyroid Nodules and Differentiated Thyroid Cancer. Thyroid 2016;26:1-133.

2. Ito $\mathrm{Y}$, Miyauchi A, Inoue H, et al. An observational trial for papillary thyroid microcarcinoma in Japanese patients. World J Surg 2010;34:28-35.

3. Ito $\mathrm{Y}$, Miyauchi A, Kihara M, et al. Patient age is significantly related to the progression of papillary microcarcinoma of the thyroid under observation. Thyroid 2014;24:27-34.

4. Wang W, Zhang Z, Zhao Y, et al. Management of Lateral Multiple-Level Metastasis in N1b Papillary Thyroid Microcarcinoma. Front Oncol 2020;10:1586.

5. Hotomi M, Sugitani I, Toda K, et al. A novel definition of extrathyroidal invasion for patients with papillary 
thyroid carcinoma for predicting prognosis. World J Surg 2012;36:1231-40.

6. Moritani S. Impact of gross extrathyroidal extension into major neck structures on the prognosis of papillary thyroid carcinoma according to the American Joint Committee on Cancer eighth edition. Endocr J 2020;67:941-8.

7. Liu Z, Huang Y, Chen S, et al. Minimal extrathyroidal extension affects the prognosis of differentiated thyroid cancer: Is there a need for change in the AJCC classification system? PLoS One 2019;14:e0218171.

8. Jung SP, Kim M, Choe JH, et al. Clinical implication of cancer adhesion in papillary thyroid carcinoma: clinicopathologic characteristics and prognosis analyzed with degree of extrathyroidal extension. World J Surg 2013;37:1606-13.

9. Podnos YD, Smith D, Wagman LD, et al. The implication of lymph node metastasis on survival in patients with well-differentiated thyroid cancer. Am Surg 2005;71:731-4.

10. Do BA, Payne RJ, Bastianelli M, et al. Is age associated with risk of malignancy in thyroid cancer? Otolaryngol Head Neck Surg 2014;151:746-50.

11. Cho JS, Yoon JH, Park MH, et al. Age and prognosis of papillary thyroid carcinoma: retrospective stratification into three groups. J Korean Surg Soc 2012;83:259-66.

12. Zanoni DK, Patel SG, Shah JP. Changes in the 8th Edition of the American Joint Committee on Cancer (AJCC) Staging of Head and Neck Cancer: Rationale and Implications. Curr Oncol Rep 2019;21:52.

13. Cho JK, Kim JY, Jeong CY, et al. Clinical features and prognostic factors in papillary thyroid microcarcinoma depends on age. J Korean Surg Soc 2012;82:281-7.

14. Roti E, degli Uberti EC, Bondanelli M, et al. Thyroid papillary microcarcinoma: a descriptive and meta-analysis study. Eur J Endocrinol 2008;159:659-73.

15. Ross DS, Litofsky D, Ain KB, et al. Recurrence after treatment of micropapillary thyroid cancer. Thyroid 2009;19:1043-8.

16. Choi Y, Jung SL. Efficacy and Safety of Thermal Ablation Techniques for the Treatment of Primary Papillary Thyroid Microcarcinoma: A Systematic Review and MetaAnalysis. Thyroid 2020;30:720-31.

17. Wang W, Bai N, Ouyang Q, et al. Prediction of level V metastases in papillary thyroid microcarcinoma: a single center analysis. Gland Surg 2020;9:899-906.

18. Ma JM, Wu LF, Hu JS, et al. Evaluation of surgical risk and prognosis between thyroid nodules of size $<1$ and $>/=1$ cm. Gland Surg 2019;8:674-82.
19. Brito JP, Ito Y, Miyauchi A, et al. A Clinical Framework to Facilitate Risk Stratification When Considering an Active Surveillance Alternative to Immediate Biopsy and Surgery in Papillary Microcarcinoma. Thyroid 2016;26:144-9.

20. Oda H, Miyauchi A, Ito Y, et al. Incidences of Unfavorable Events in the Management of Low-Risk Papillary Microcarcinoma of the Thyroid by Active Surveillance Versus Immediate Surgery. Thyroid 2016;26:150-5.

21. Wang W, Yang Z, Ouyang Q. A nomogram to predict skip metastasis in papillary thyroid cancer. World J Surg Oncol 2020;18:167.

22. Cibas ES, Ali SZ. The Bethesda System for Reporting Thyroid Cytopathology. Thyroid 2009;19:1159-65.

23. Kwak JY, Han KH, Yoon JH, et al. Thyroid imaging reporting and data system for US features of nodules: a step in establishing better stratification of cancer risk. Radiology 2011;260:892-9.

24. Adam MA, Pura J, Goffredo P, et al. Presence and Number of Lymph Node Metastases Are Associated With Compromised Survival for Patients Younger Than Age 45 Years With Papillary Thyroid Cancer. J Clin Oncol 2015;33:2370-5.

25. Zhang D, Tang J, Kong D, et al. Impact of Gender and Age on the Prognosis of Differentiated Thyroid Carcinoma: a Retrospective Analysis Based on SEER. Horm Cancer 2018;9:361-70.

26. Kandil E, Noureldine SI, Tufano RP. Thyroidectomy vs Active Surveillance for Subcentimeter Papillary Thyroid Cancers--The Cost Conundrum. JAMA Otolaryngol Head Neck Surg 2016;142:9-10.

27. Bolfi F, Domingues MA, Sobrinho-Simoes M, et al. Primary squamous cell carcinoma of the thyroid diagnosed as anaplastic carcinoma: failure in fine-needle aspiration cytology? Case Rep Pathol 2014;2014:301780.

28. Wang W, Ouyang Q, Meng C, et al. Treatment optimization and prognostic considerations for primary squamous cell carcinoma of the thyroid. Gland Surg 2019;8:683-90.

29. Abraham E, Roshan D, Tran B, et al. The extent of extrathyroidal extension is a key determinant of prognosis in T4a papillary thyroid cancer. J Surg Oncol 2019;120:1016-22.

30. Lee YK, Kim D, Shin DY, et al. The Prognosis of Papillary Thyroid Cancer with Initial Distant Metastasis is Strongly Associated with Extensive Extrathyroidal Extension: A Retrospective Cohort Study. Ann Surg Oncol 2019;26:2200-9. 
31. Zaydfudim V, Feurer ID, Griffin MR, et al. The impact of lymph node involvement on survival in patients with papillary and follicular thyroid carcinoma. Surgery 2008;144:1070-7; discussion 1077-8.

Cite this article as: Meng C, Wang W, Zhang Y, Li X. The influence of nodule size on the aggressiveness of thyroid carcinoma varies with patient's age. Gland Surg 2021;10(3):961972. doi: $10.21037 / \mathrm{gs}-20-747$
32. Ito Y, Miyauchi A, Kobayashi K, et al. Prognosis and growth activity depend on patient age in clinical and subclinical papillary thyroid carcinoma. Endocr J 2014;61:205-13. 